\title{
Instabilidade multidirecional em uma luxação negligenciada do cotovelo gerenciada com reconstrução do ligamento circunferencial. Um relato de caso*
}

\section{Multidirectional Instability in a Neglected Elbow Dislocation Managed with Circumferential Ligament Reconstruction. A Case Report}

\author{
Sathya Vamsi Krishna ${ }^{10}$ \\ Madan Ballal2@ \\ ${ }^{1}$ Departamento de Cirurgia da Mão, Instituto de Ortopedia e Trauma \\ Sanjay Gandhi, Bangalore, Karnataka, Índia \\ 2 Departamento de Ortopedia e Lesões Esportivas, Instituto de \\ Ortopedia e Trauma Sanjay Gandhi, Karnataka, Índia
}

\author{
Vivekanandea $B R^{2}$
}

Endereço para correspondência Sathya Vamsi Krishna, MBBS, MS, Department of Hand Surgery, Sanjay Gandhi Institute of Orthopaedics and Trauma, Byrasandra, Jayanagar, Bangalore, Karnataka, India (e-mail: sathyavamsi@yahoo.com).

Rev Bras Ortop 2023;58(1):168-172.

\section{Resumo \\ Palavras-chave \\ - articulação do cotovelo \\ - luxação articular \\ - instabilidade articular \\ - ligamentos articulares}

A luxação negligenciada do cotovelo é uma condição incomum e seu tratamento permanece desafiador. Apresentamos o caso de uma mulher de meia-idade que apresentou luxação negligenciada do cotovelo e instabilidade multidirecional, na qual foi realizada redução aberta da articulação do cotovelo e reconstrução ligamentar circunferencial com enxerto de tendão gracilis. O resultado funcional avaliado com o índice de desempenho do cotovelo de Mayo foi excelente. Essa técnica circunferencial é, sem dúvida, uma técnica viável e as indicações podem ser estendidas para gerenciar até mesmo um deslocamento negligenciado. Este procedimento reduz a necessidade ou diminui a duração da exigência de fixação externa e, assim, incentiva a mobilização precoce.

Neglected elbow dislocation is an uncommon condition and its treatment remains challenging. We present a case of a middle-aged woman presenting with neglected elbow dislocation and multi-direction instability in whom open reduction of the elbow joint and circumferential ligamentous reconstruction with a gracilis tendon graft was done. The functional outcome assessed with the Mayo elbow performance index was excellent. This circumferential technique is undoubtedly a viable technique and the indications can be extended to even manage a neglected dislocation. This procedure reduces the need or diminishes the duration of external fixation requirement and thereby encourages early mobilization.

\footnotetext{
Estudo realizado no Departamento de Cirurgia da Mão, Instituto de Ortopedia e Trauma Sanjay Gandhi, Byrasandra, Jayanagar, Bangalore, Índia.
}

recebido 30 de Março de 2020 aceito

01 de Junho de 2020 article Publicado on-line Dezembro 25, 2020
DOI https://doi.org/ 10.1055/s-0040-1714232. ISSN 0102-3616.
(C) 2020. Sociedade Brasileira de Ortopedia e Traumatologia. All rights reserved.

This is an open access article published by Thieme under the terms of the Creative Commons Attribution-NonDerivative-NonCommercial-License, permitting copying and reproduction so long as the original work is given appropriate credit. Contents may not be used for commercial purposes, or adapted, remixed, transformed or built upon. (https://creativecommons.org/ licenses/by-nc-nd/4.0/) Thieme Revinter Publicações Ltda., Rua do Matoso 170, Rio de Janeiro, RJ, CEP 20270-135, Brazil 


\section{Introdução}

As luxações do cotovelo são as segundas luxações mais comuns no membro superior, logo após as de ombro, e as luxações simples do cotovelo têm bons prognósticos com redução fechada. No entanto, as luxações negligenciadas do cotovelo, embora incomuns, são um desafio para o tratamento, e as opções de tratamento incluem redução aberta, reparo/reconstrução ligamentar, aplicação de fixador externo e artroplastia do cotovelo, considerando a duração da lesão e o estado articular. Embora tecnicamente exigente, um procedimento de estágio único para reduzir e reconstruir o cotovelo deslocado negligenciado seria ideal para permitir o retorno precoce das funções e evitar instabilidade.

No presente artigo, descrevemos um paciente que apresentou uma luxação mediano-posterior negligenciada por 3 meses com instabilidade multidirecional/global, tratado com redução aberta e reconstrução ligamentar colateral com autoenxerto do gracilis usando a técnica de reconstrução do ligamento circunferencial. ${ }^{1}$

\section{Relato de Caso}

Uma mulher de 30 anos, dona de casa, apresentou queixas de dor no cotovelo direito e instabilidade após um histórico de queda acidental no trânsito 3 meses antes e lesão no membro superior direito. Inicialmente, foi diagnosticada fratura do rádio distal direito, pela qual foi submetida a uma redução aberta e fixação com placa. No entanto, ela persistiu com dor no cotovelo direito após 3 meses e veio até nós. 0 cotovelo estava frouxo, com instabilidade multidirecional associada à dor e ao estalo. As radiografias do cotovelo revelaram luxação medial-posterior do cotovelo sem fraturas (-Fig. 1). A tomografia computadorizada (TC) e a ressonância magnética (RM) mostraram luxação com lesão do complexo do ligamento colateral lateral ulnar (LCLU) e nenhum sinal de artrose. Após o consentimento, foi encaminhada para redução aberta e reconstrução ligamentar.

Sob anestesia geral, inicialmente o tendão de gracilis foi colhido do membro inferior. Em seguida, a paciente foi posicionada em decúbito lateral com o braço apoiado em um aparador. A técnica cirúrgica de redução é semelhante à mencionada por Morrey, ${ }^{2}$ e para reconstrução ligamentar, a técnica de alça única de van Riet et al. ${ }^{1}$ foi adotada com certas modificações ( - Figs. 2 e 3). Sob controle de torniquete, incisão universal posterior da linha média foi feita no cotovelo e retalhos fasciocutâneos foram elevados em ambos os lados. O nervo ulnar foi protegido e, elevando o músculo flexor ulnar do carpo, o epicôndilo medial e o tubérculo sublime da ulna foram expostos. No lado radial, o epicôndilo lateral e a crista do supinador foram expostos pela abordagem de Kocher. Após a liberação das colaterais cicatrizadas e apertadas, da cápsula anterior e posterior do lado umeral, conseguimos realizar a redução da articulação. A superfície articular era saudável, mas instável. Para proporcionar estabilidade à articulação reduzida, procedemos à reconstrução do feixe posterior do LCLU e do feixe anterior do ligamento colateral medial (LCM). Primeiro, um fio guia foi passado na região intercondilar do úmero distal através do eixo de rotação e sobreperfurado com broca de $4,5 \mathrm{~mm}$. Dois furos foram feitos na ulna proximal na crista do supinador e no tubérculo sublime direcionado distalmente e perfurando obliquamente os córtices opostos. Um ponto em chicote foi aplicado na extremidade inicial e um Endobutton (Smith \& Nephew, Londres, Inglaterra) foi ancorado na extremidade posterior do enxerto gracilis usando material de sutura Ethibond no.5 (Ethicon Inc., Sommerville, NJ, EUA). A extremidade anterior do enxerto foi passada através do túnel na crista do supinador e o enxerto foi avançado através do túnel do úmero distal do lado lateral para o medial que formava o LCLU. Aqui o enxerto foi tensionado e fixado inserindo um parafuso de interferência no túnel do úmero, mantendo o cotovelo em pronação. Então o enxerto trazido pelo lado medial do túnel do úmero foi passado e fixado no túnel ulnar no tubérculo sublime com outro parafuso de interferência que mantinha o cotovelo em flexão. A amplitude de movimento livre e a estabilidade foram satisfatórias. 0 excesso de enxerto foi excisado e a pele foi fechada em camadas. $O$ cotovelo foi imobilizado com tala acima do cotovelo por 3 semanas, iniciando na amplitude de movimento do cotovelo com a tipoia do braço continuada por 6 semanas. A paciente não apresentou complicações relacionadas à cicatrização de nervos e feridas. Dentro de 6 semanas, ela conseguiu

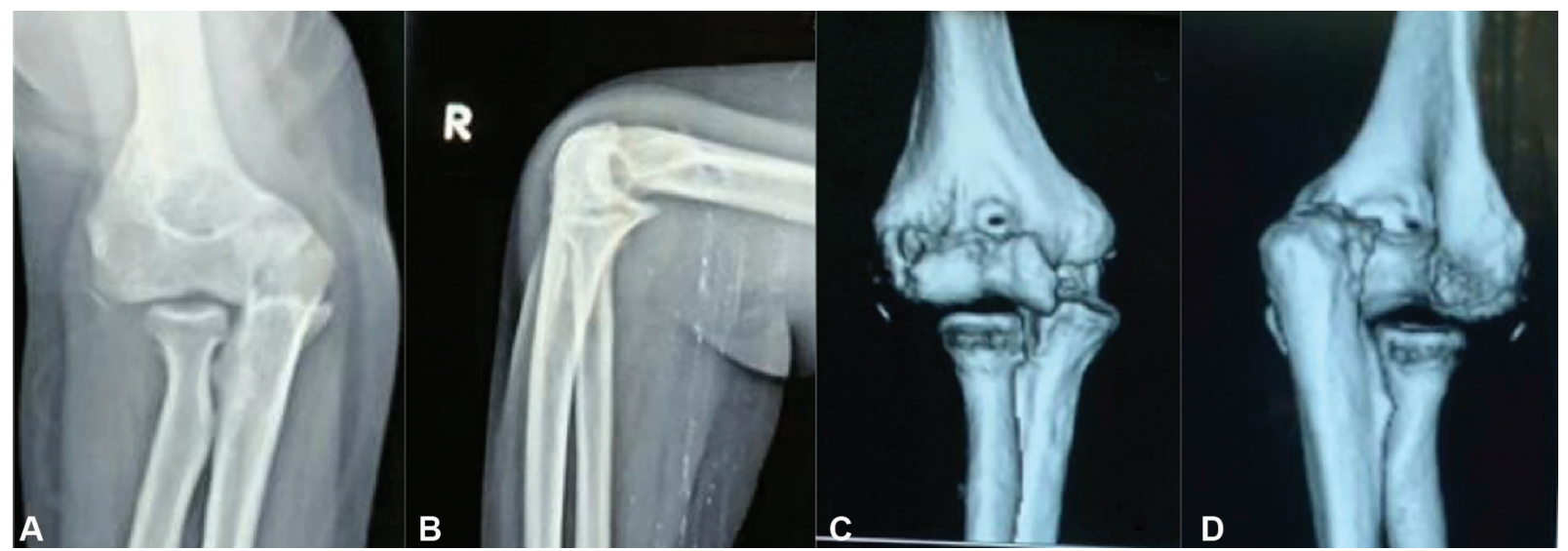

Fig. 1 (A, B) Radiografia plana da articulação afetada mostrando luxação mediano-posterior negligenciada do cotovelo; (C, D) Tomografia computadorizada (TC) imagens do cotovelo deslocado revelando um cotovelo deslocado sem fraturas óbvias. 

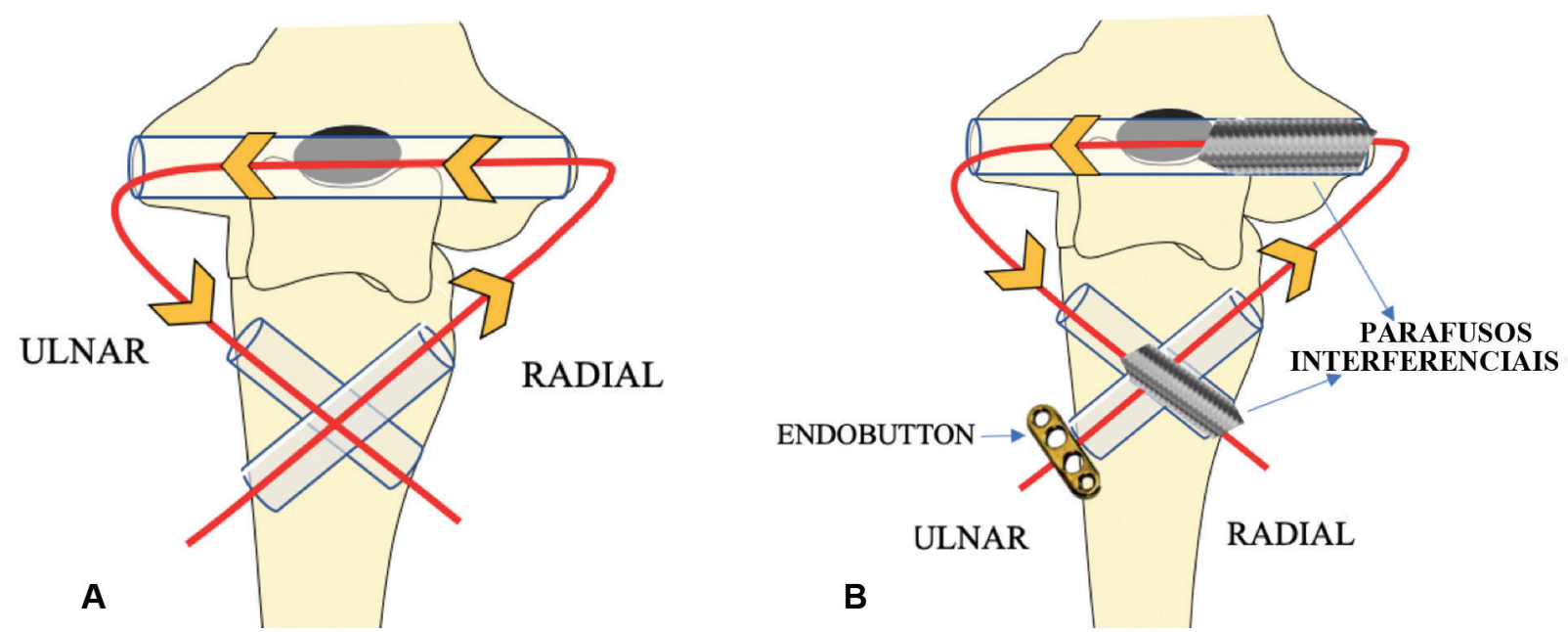

Fig. 2 Representação gráfica da técnica cirúrgica para reconstrução em alça única do complexo ligamentar medial e lateral do cotovelo. AForam criados três túneis ósseos, um no úmero distal e dois na ulna proximal no tubérculo sublime e na crista do supinador. O enxerto é tunelizado através da crista do supinador e avançado através do túnel do úmero para reconstruir o feixe posterior de LCLU e retornado ao túnel no tubérculo sublime para formar o feixe anterior do LCM. B- A extremidade posterior do enxerto na ulna é fixada por um Endobutton (Smith \& Nephew, Londres, Inglaterra); O LCLU no túnel umeral e o LCM no túnel ulnar no tubérculo sublime foram fixados e tensionados com parafusos de interferência (LCLU - Ligamento colateral lateral ulnar, LCM- Ligamento colateral medial).
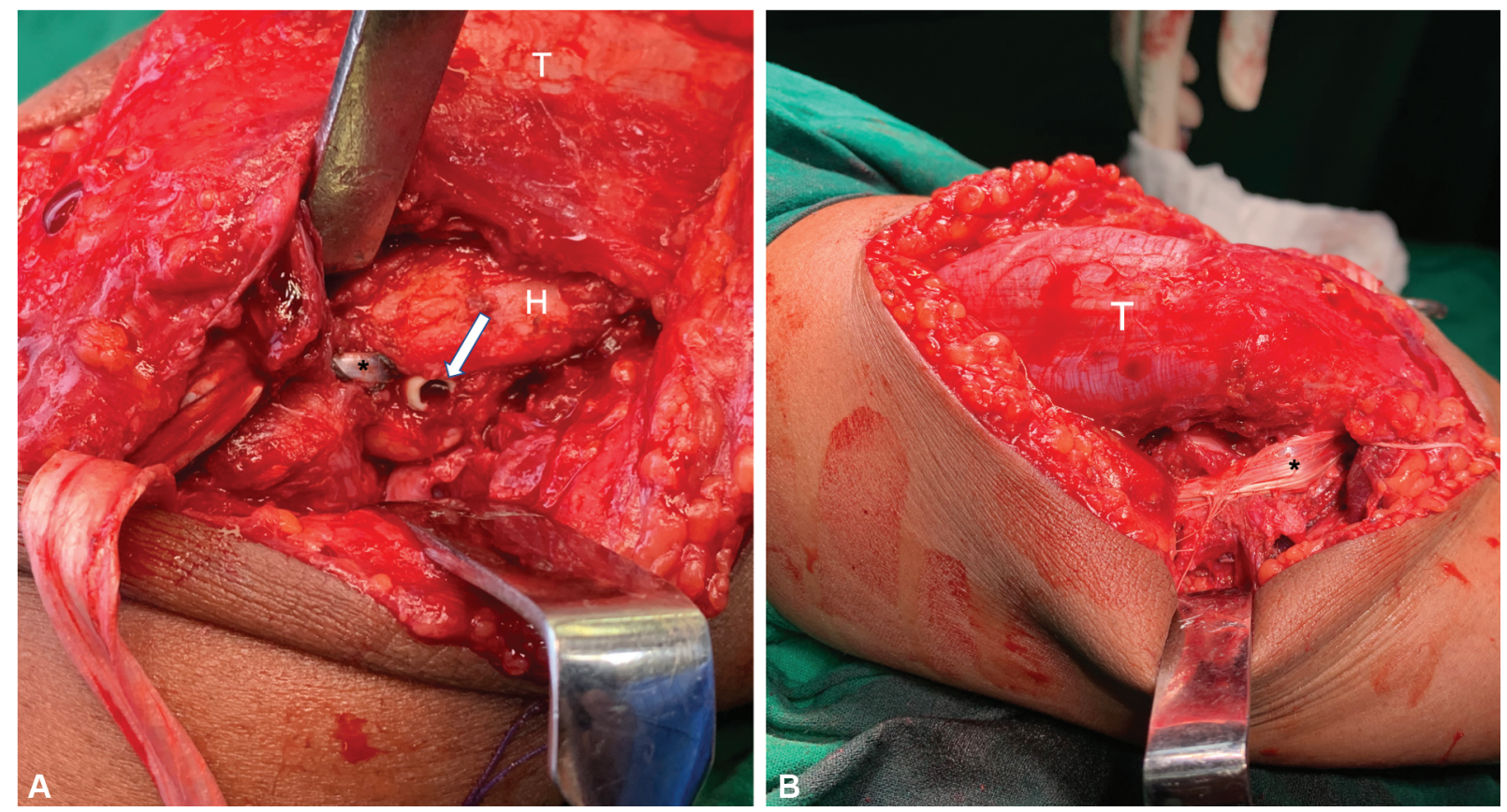

Fig. 3 Nas imagens intraoperatórias, o cotovelo está colocado em posição de decúbito lateral. A- Posição do parafuso de interferência na pegada do complexo ligamentar lateral ulnar no úmero distal juntamente com o enxerto tensionado. B- Enxerto de ligamento colateral medial tensionado passando da pegada do úmero distal para o tubérculo sublime da ulna. (T- tríceps, H- úmero, * - Enxerto do Grácil/Gracilis).

atingir uma amplitude de $10^{\circ}$ a $130^{\circ}$ de movimento. No acompanhamento de 1 ano, o resultado funcional foi avaliado com base no índice de desempenho do cotovelo de Mayo, ${ }^{3}$ que considera parâmetros de dor, movimento, estabilidade e funções. Ela teve excelente resultado e as radiografias mostraram

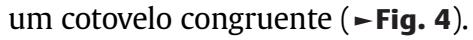

\section{Discussão}

A estabilidade da articulação do cotovelo é fornecida pelas articulações ulnoumeral e radiocapitelar, além do LCLU e do
LCM. O feixe anterior do LCM desempenha um papel central, proporcionando estabilidade ao estresse em valgo ${ }^{4}$ e o LCLU fornece estabilidade posterolateral. A maioria das luxações simples do cotovelo cicatriza bem após a redução, sem nenhuma intervenção e com a apropriada mobilização. ${ }^{5} \mathrm{O}$ cotovelo deslocado cronicamente instável ou deslocado é o resultado de lesões inadequadamente tratadas da cabeça radial, coronoide ou ligamentos. ${ }^{6}$ Os sintomas variam de instabilidade leve a grave, limitando as atividades dos membros superiores, subluxação ou luxação recorrente/fixa. Isso pode causar cliques dolorosos, estalos, batidas ou travamento do cotovelo. ${ }^{3}$ Se negligenciado, 


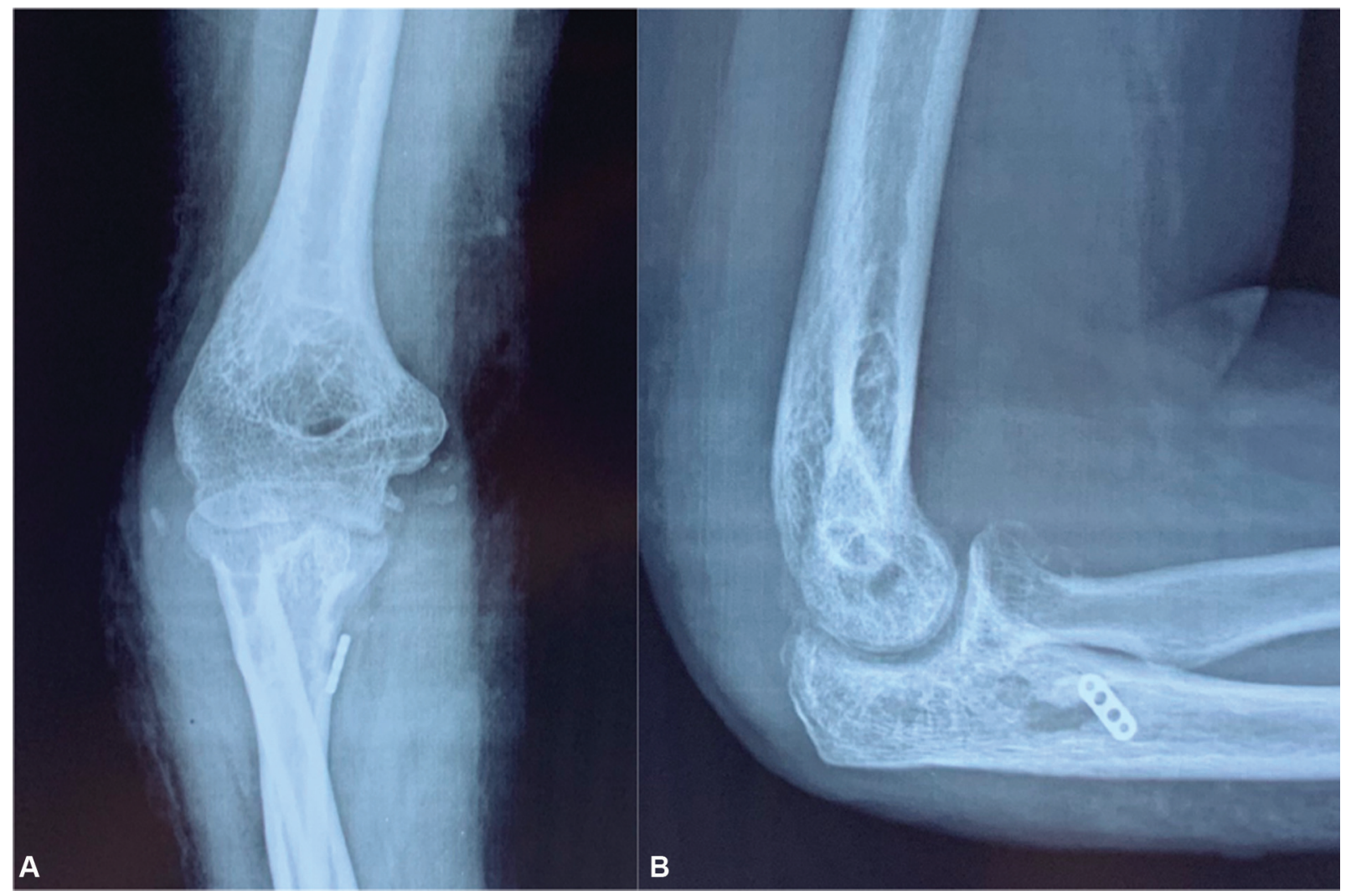

Fig. 4 (A, B) Radiografias de acompanhamento mostrando articulação do cotovelo reduzida e congruente, sem sinais de artrose.

pode levar à artrite ulnoumeral. A redução fechada torna-se difícil por causa de fibrose na articulação ulnoumeral, contraturas do tríceps e ligamentos colaterais e possíveis ossificações heterotrópicas. ${ }^{7,8}$ Se a redução exigir a liberação de tecidos moles, é necessária a reconstrução definitiva dos ligamentos colaterais. ${ }^{6}$ Os resultados são melhores com a redução aberta dentro de 3 a 6 meses após a luxação, ou então uma artroplastia deveria ser considerada. ${ }^{8}$ Essa luxação negligenciada precisa de redução aberta e fixadores externos articulados, permitindo que estruturas capsuloligamentares sarem ou formem uma cicatriz estável. Lesões crônicas de LCLU e LCM com instabilidades podem ser reconstruídas usando enxertos de palmar longo, plantar e fáscia do tríceps. ${ }^{9-11}$

Em seu trabalho, van Riet et al. ${ }^{1}$ demonstrou uma técnica usando um único tendão gracilis, que é novamente dividido em dois tipos, alça única ou alças duplas, com base no número de feixes de LCLU e LCM reconstruídos. ${ }^{1}$ A modificação do design conhecido como "loop de caixa" é descrita sem o uso de outros materiais. ${ }^{12}$ Um estudo in vitro, feito para comparar a técnica de "loop de caixa" com a reconstrução convencional do LCLU e do LCM, descobriu que ambos tinham estabilidade igual; no entanto, a técnica anterior tinha um perfil biomecânico superior em relação à rigidez do enxerto. ${ }^{13}$ Usamos a técnica de loop único para substituir estruturas críticas. Embora a técnica original recomende que a mobilização precoce seja de 1 semana, imobilizamos por 3 semanas para a cura do ligamento. A maior parte da literatura descreve o uso da técnica de enxerto circunferencial em instabilidade crônica secundária a vários tipos de lesões, mas, de acordo com o nosso conhecimento, essa técnica não foi usada para uma luxação negligenciada do cotovelo. A técnica circunferencial de enxerto é um método viável no tratamento de instabilidades crônicas do cotovelo com um único enxerto. Suas indicações podem ser estendidas para tratar até a luxação negligenciada do cotovelo e reduzem a necessidade ou diminuem a duração da exigência de fixação externa, desde que o enxerto seja tensionado adequadamente.

Declaração de Consentimento Informado

A paciente foi informada de que os dados referentes ao caso seriam submetidos à publicação, com o que a paciente concordou.

\section{Conflito de Interesses}

Os autores declaram não haver conflito de interesses.

\section{Agradecimentos}

Nenhum financiamento externo foi recebido para nenhum aspecto deste trabalho. Gostaríamos de agradecer o apoio do Prof. van Riet e do Dr. David Tan na execução do procedimento. Os autores também gostariam de agradecer ao Dr. Chandrashekar H. S. e ao Sr. Palanivel.

\section{Referências}

1 van Riet RP, Bain GI, Baird R, Lim YW. Simultaneous reconstruction of medial and lateral elbow ligaments for instability using a circumferential graft. Tech Hand Up Extrem Surg 2006;10(04): 239-244 
2 Morrey BF. Chronic Unreduced Elbow Dislocation. Elb Its Disord. 4th ed. Philadelphia, PA: Saunders WB; 2008:463-471

3 Morrey BF. Functional evaluation of elbow. Elb Its Disord. 4th ed. Philadelphia, PA: Saunders WB; 2008:88

4 Morrey BF, Tanaka S, An KN. Valgus stability of the elbow. A definition of primary and secondary constraints. Clin Orthop Relat Res 1991;(265):187-195

5 van der Ley J, van Niekerk JL, Binnendijk B. Conservative treatment of elbow dislocations in adults. Neth J Surg 1987;39(06): 167-169

6 Lyons RP, Armstrong A. Chronically unreduced elbow dislocations. Hand Clin 2008;24(01):91-103

7 di Schino M, Breda Y, Grimaldi FM, Lorthioir JM, Merrien Y. [Surgical treatment of neglected elbow dislocations. Report of 81 cases]. Rev Chir Orthop Repar Appar Mot 1990;76(05):303-310
8 Silva JF. Old dislocations of the elbow. Ann R Coll Surg Engl 1958; 22(06):363-381

9 Nestor BJ, O'Driscoll SW, Morrey BF. Ligamentous reconstruction for posterolateral rotatory instability of the elbow. J Bone Joint Surg Am 1992;74(08):1235-1241

10 Sanchez-Sotelo J, Morrey BF, O'Driscoll SW. Ligamentous repair and reconstruction for posterolateral rotatory instability of the elbow. J Bone Joint Surg Br 2005;87(01):54-61

11 Eygendaal D. Ligamentous reconstruction around the elbow using triceps tendon. Acta Orthop Scand 2004;75(05):516-523

12 Finkbone PR, O'Driscoll SW. Box-loop ligament reconstruction of the elbow for medial and lateral instability. J Shoulder Elbow Surg 2015;24(04):647-654

13 Hackl M, Leschinger T, Müller LP, Wegmann K. [Chronic ligamentous instability of the elbow]. Orthopade 2016;45(10):809-821 